Pacific Journal of Mathematics

MEAN CROSS-SECTION MEASURES OF HARMONIC MEANS

WI LIAM JAMES Fine Y 


\title{
MEAN CROSS-SECTION MEASURES OF HARMONIC MEANS OF CONVEX BODIES
}

\author{
WILLIAM J. FIREY
}

1. In [2] the notion of $p$-dot means of two convex bodies in Euclidean $n$-space was introduced and certain properties of these means investigated. For $p=1$, the mean is more appropriately called the harmonic mean; here we restrict the discussion to this case. The harmonic mean of two convex bodies $K_{0}$ and $K_{1}$, which will always be assumed to share a common interior point $Q$, is defined as follows. Let $\hat{K}$ denote the polar reciprocal of $K$ with respect to the unit sphere $E$ centred at $Q$; let $(1-\vartheta) \widehat{K}_{0}+\vartheta \hat{K}_{1}$, with $0 \leqq \vartheta \leqq 1$, be the usual arithmetic or Minkowski mean of $\hat{K}_{0}$ and $\hat{K}_{1}$. The harmonic mean of $K_{0}, K_{1}$ is the convex body $\left[(1-\vartheta) \hat{K}_{0}+\vartheta \hat{K}_{1}\right]^{\wedge}$. In more analytic terms, if $F_{i}(x)$ are the distance functions with respect to $Q$ of $K_{i}$, for $i=0,1$, then the body whose distance function with respect to $Q$ is $(1-\vartheta) F_{0}(x)+\vartheta F_{1}(x)$ is the harmonic mean of $K_{0}$ and $K_{1}$.

In the paper mentioned, a dual Brunn-Minkowski theorem was established, namely

$$
V^{1 / n}\left(\left[(1-\vartheta) \hat{K}_{0}+\vartheta \hat{K}_{1}\right]^{\wedge}\right) \leqq 1 /\left[\frac{(1-\vartheta)}{V^{1 / n}\left(K_{0}\right)}+\frac{\vartheta}{V^{1 / n}\left(K_{1}\right)}\right]
$$

where $V(K)$ means the volume of $K$. There is equality if and only if $K_{0}$ and $K_{1}$ are homothetic with the centre of magnification at $Q$.

Here we develop a more inclusive theorem regarding the behaviour of each mean cross-section measure, ("Quermassintegral") $W_{\nu}(K), \nu=$ $0,1, \cdots, n-1$, cf. [1]. The result is

$$
W_{\nu}^{1 /(n-\nu)}\left(\left[(1-\vartheta) \hat{K}_{0}+\vartheta \hat{K}_{1}\right]^{\wedge}\right) \leqq 1 /\left[\frac{(1-\vartheta)}{W_{\nu}^{1 /(n-\nu)}\left(K_{0}\right)}+\frac{\vartheta}{W_{\nu}^{1 /(n-\nu)}\left(K_{1}\right)}\right] .
$$

The cases of equality are just those of the dual Brunn-Minkowski theorem, $(\nu=0)$.

2. We first list some preliminary items used in the proof of (2). We shall use Minkowski's inequality in the form

$$
\int\left[(1-\vartheta) f_{0}^{p}+\vartheta f_{1}^{p}\right]^{1 / p} d x \leqq\left[(1-\vartheta)\left(\int f_{0} d x\right)^{p}+\vartheta\left(\int f_{1} d x\right)^{p}\right]^{1 / p} .
$$

Here the functions $f_{i}$ are assumed to be positive and continuous over the closed and bounded domain of integration common to all the integrals,

Received September 29, 1960. 
and, for our puposes, $p$ satisfies $-1 \leqq p<0$. There is equality if and only if $f_{0}(x) \equiv \lambda f_{1}(x)$ for some constant $\lambda$. See [3], Theorem 201, coupled with the remark preceding Theorem 200.

Our second tool, which we shall refer to as the projection lemma, was established in [2]. Let $K^{*}$ denote the projection of $K$ onto a fixed, $m$-dimensional, linear subspace $E_{m}$ through $Q$ for $1 \leqq m<n$. We have

$$
\left[(1-\vartheta) \hat{K}_{0}^{*}+\vartheta \hat{K}_{1}^{*}\right]^{\wedge} \supseteqq\left\{\left[(1-\vartheta) \hat{K}_{0}+\vartheta \hat{K}_{1}\right]^{\wedge}\right\}^{*} .
$$

Since $E_{m}$ contains $Q$ and the polar reciprocation is with respect to sphere $E$ centred at $Q$, in forming $\hat{K}^{*}$ the order of operations is immaterial. This result is proved by a polar reciprocation argument from

$$
(1-\vartheta)\left(\hat{K}_{0} \cap E_{m}\right)+\vartheta\left(\hat{K}_{1} \cap E_{m}\right) \subseteq\left[(1-\vartheta) \hat{K}_{0}+\vartheta \hat{K}_{1}\right] \cap E_{m} .
$$

There is equality in either inclusion if $K_{0}$ and $K_{1}$ are homothetic with centre of magnification at $Q$.

The dual Brunn-Minkowski theorem (1) will be used.

Finally we shall make use of Kubota's formula and some of its consequences. This material is covered in [1]. An $(n-\nu)$ dimensional cross-section measure ("Quermass") of $K$ is the $(n-\nu)$ dimensional volume of that convex body which is the vertical projection of $K$ onto an $E_{n-\nu}$. The mean cross-section measures are usually defined as the coefficients in Steiner's polynomial which describes $V(K+\lambda E)$, that is

$$
V(K+\lambda E)=\sum_{\nu=0}^{n}\left(\begin{array}{l}
n \\
\nu
\end{array}\right) W_{\nu}(K) \lambda^{\nu} .
$$

If we denote the $(\nu-1)^{\text {th }}$ mean cross-section measure of the projection of $K$ onto that $E_{n-1}$ through $Q$ which is orthogonal to the vector $u_{1}$ by $W_{\nu-1}^{\prime}\left(K, u_{1}\right)$, then Kubota's formula is

$$
W_{\nu}(K)=\frac{1}{\kappa_{n-1}} \int_{\Omega_{n}} W_{\nu-1}^{\prime}\left(K, u_{1}\right) d \omega_{n}, \quad \nu=1,2, \cdots, \nu-1 .
$$

Here the integration with respect to the direction $u_{1}$ is extended over the surface $\Omega_{n}$ of $E, d \omega_{n}$ is the element of surface area on $\Omega_{n}$ and $\kappa_{n-1}$ is the volume of the $n-1$ dimensional unit sphere.

Kubota's formula can be applied to the mean cross-section measure $W_{\nu-1}^{\prime}\left(K, u_{1}\right)$ for fixed $u_{1}$ :

$$
W_{\nu-1}^{\prime}\left(K, u_{1}\right)=\frac{1}{\kappa_{n-2}} \int_{\Omega_{n-1}} W_{\nu-2}^{\prime \prime}\left(K, u_{1}, u_{2}\right) d \omega_{n-1}
$$

where $W_{\nu-2}^{\prime \prime}$ is the $(\nu-2)$ th mean cross-section measure of the projection of $\kappa$ onto the $E_{n-2}$ through $Q$ orthogonal to $u_{1}$ and $u_{2}$ with $u_{2}$ orthogonal to $u_{1}$. After $\nu$ such steps we have as the extended form of Kubota's formula: 
$W_{\nu}(K)$

$=\frac{1}{\kappa_{n-1} \kappa_{n-2} \cdots \kappa_{n-\nu}} \int_{\Omega_{n}} \int_{\Omega_{n-1}} \cdots \int_{\Omega_{n-\nu}} W_{0}^{(\nu)}\left(K, u_{1}, u_{2}, \cdots, u_{\nu}\right) d \omega_{n-\nu} \cdots d \omega_{n-1} d \omega_{n}$.

Each vector $u_{p}$ is orthogonal to $u_{q}$ for $q<p$ and $W_{0}^{(\nu)}\left(K, u_{1}, u_{2}, \cdots, u_{\nu}\right)$ is the 0th mean cross-section measure of the projection of $K$ onto that $E_{n-\nu}$ through $Q$ which is the orthogonal complement of the subspace spanned by $u_{1}, u_{2}, \cdots, u_{\nu}$.

Steiner's formula (5) with $\lambda=0$ shows that $W_{0}(K)$ is the volume of $K$ and so $W_{0}^{(\nu)}$ is an $(n-\nu)$ dimensional cross-section measure of $K$. Thus, to within a numerical factor depending on $n$ and $\nu, W_{\nu}(K)$ is the arithmetic mean of the $(n-\nu)$ dimensional cross-section measures.

In $\S 3$ we shall use the following abbreviations: for $d \omega_{n-\nu} \cdots d \omega_{n-1} d \omega_{n}$ we write $d \bar{\omega}$ with sign of integration and omit reference to the domains of integration; for one $1 / \kappa_{n-1} \kappa_{n-2} \cdots \kappa_{n-\nu}$ we write $k$; finally for $W_{0}^{(\nu)}\left(K, u_{1}\right.$, $u_{2}, \cdots, u_{\nu}$ ) we write $\sigma\left(K^{*}\right)$. In this notation the extended Kubota formula reads

$$
W(K)=k \int \sigma\left(K^{*}\right) d \bar{\omega}
$$

3. We now prove (2). By the extended form of Kubota's formula

$$
\begin{aligned}
W_{\nu}^{1 /(n-\nu)}\left(\left[(1-\vartheta) \hat{K}_{0}+\vartheta \hat{K}_{1}\right]^{\wedge}\right) & =\left[k \int \sigma\left(\left\{\left[(1-\vartheta) \hat{K}_{0}+\vartheta \hat{K}_{1}\right]^{\wedge}\right\}^{*}\right) d \bar{\omega}\right]^{1 /(n-\nu)} \\
& \leqq\left[k \int \sigma\left(\left[(1-\vartheta) \hat{K}_{0}^{*}+\vartheta \hat{K}_{1}^{*}\right]^{\wedge}\right) d \bar{\omega}\right]^{1 /(n-\nu)}
\end{aligned}
$$

in virtue of the projection lemma and the set monotonicity of $\sigma$ i.e., $\sigma\left(K^{*}\right) \leqq \sigma\left(\bar{K}^{*}\right)$ if $K^{*} \subseteq \bar{K}^{*}$ with equality in the latter relation implying that in the former. We now apply (1), in $E_{n-\nu}$, to the integrand to obtain

$$
\sigma\left(\left[(1-\vartheta) \hat{K}_{0}^{*}+\vartheta \hat{K}_{1}^{*}\right]^{\wedge}\right) \leqq\left\{1 /\left[\frac{(1-\vartheta)}{\sigma^{1 /(n-\nu)}\left(K_{0}^{*}\right)}+\frac{\vartheta}{\sigma^{1 /(n-\nu)}\left(K_{1}^{*}\right)}\right]\right\}^{(n-\nu)} .
$$

Here we take advantage of the fact that

$$
(\hat{K})^{*}=\left(K^{*}\right)^{\wedge} \text {. }
$$

This gives

$$
\begin{aligned}
& W_{\nu}^{1 /(n-\nu)}\left(\left[(1-\vartheta) \hat{K}_{0}+\vartheta \hat{K}_{1}\right]^{\wedge}\right) \\
& \quad \leqq\left[k \int\left\{1 /\left[\frac{(1-\vartheta)}{\sigma^{1 /(n-\nu)}\left(K_{0}^{*}\right)}+\frac{\vartheta}{\sigma^{1 /(n-\nu)}\left(K_{1}^{*}\right)}\right]\right\}^{(n-\nu)} d \bar{\omega}\right]^{1 /(n-\nu)} .
\end{aligned}
$$

There is equality if and only if all the projections $K_{0}^{*}$ and $K_{1}^{*}$ are homothetic with the centre of magnification at $Q$. This condition is 
sufficient for equality in (6); it is necessary and sufficient for (7). yields

We now use Minkowski's inequality (3) with $p=-1 / n-\nu$. This

$$
\begin{aligned}
& W_{\nu}^{1 /(n-\nu)}\left(\left([1-\vartheta) \hat{K}_{0}+\vartheta \hat{K}_{1}\right)\right. \\
& \quad \leqq 1 /\left[\frac{(1-\vartheta)}{\left(k \int \sigma\left(K_{0}^{*}\right) d \bar{\omega}\right)^{1 /(n-\nu)}}+\frac{\vartheta}{\left(k \int \sigma\left(K_{1}^{*}\right) d \bar{\omega}\right)^{1 /(n-\nu)}}\right] \\
& \quad=1 /\left[\frac{(1-\vartheta)}{W_{\nu}^{1 /(n-\nu)}\left(K_{0}\right)}+\frac{\vartheta}{W_{\nu}^{1 /(n-\nu)}\left(K_{1}\right)}\right] .
\end{aligned}
$$

The necessary and sufficient conditions for equality in (7) are sufficient for equality in (3) since $K_{0}=\lambda K_{1}$ implies $\sigma\left(K_{0}^{*}\right)=\lambda^{n-\nu} \sigma\left(K_{1}^{*}\right)$. This establishes (2).

\section{REFERENCES}

1. T. Bonnesen and W. Fenchel, Konvexe Körper, Berlin, 1934, reprint N. Y. (1948), 48-50. 2. W. J. Firey, Polar Means of Convex Bodies and a Dual to the Brunn-Minkowski theorem. Canadian Math. J., 13 (1961), 444-453.

3. G. Hardy, J. Littlewood, and G. Pólya, Inequalities, Cambridge, (1934), 148.

WASHINGTON State UNIVERSity 


\section{PACIFIC JOURNAL OF MATHEMATICS}

\section{EDITORS}

\author{
RaLPh S. Phillips \\ Stanford University \\ Stanford, California \\ F. H. BRowNELL \\ University of Washington \\ Seattle 5 , Washington
}

A. L. Whiteman

University of Southern California

Los Angeles 7, California

L. J. Paige

University of California

Los Angeles 24, California

\author{
E. F. BECKENBACH \\ T. M. CHERRY
}

\author{
ASSOCIATE EDITORS

$\begin{array}{lll}\text { D. DERRY } & \text { H. L. ROYDEN } & \text { E. G. STRAUS } \\ \text { M. OHTSUKA } & \text { E. SPANIER } & \text { F. WOLF }\end{array}$

\section{SUPPORTING INSTITUTIONS}

\author{
UNIVERSITY OF BRITISH COLUMBIA \\ CALIFORNIA INSTITUTE OF TECHNOLOGY \\ UNIVERSITY OF CALIFORNIA \\ MONTANA STATE UNIVERSITY \\ UNIVERSITY OF NEVADA \\ NEW MEXICO STATE UNIVERSITY \\ OREGON STATE COLLEGE \\ UNIVERSITY OF OREGON \\ OSAKA UNIVERSITY \\ UNIVERSITY OF SOUTHERN CALIFORNIA
}

\author{
STANFORD UNIVERSITY \\ UNIVERSITY OF TOKYO \\ UNIVERSITY OF UTAH \\ WASHINGTON STATE COLLEGE \\ UNIVERSITY OF WASHINGTON \\ AMERICAN MATHEMATICAL SOCIETY \\ CALIFORNIA RESEARCH CORPORATION \\ HUGHES AIRCRAFT COMPANY \\ SPACE TECHNOLOGY LABORATORIES \\ NAVAL ORDNANCE TEST STATION
}

Mathematical papers intended for publication in the Pacific Journal of Mathematics should be typewritten (double spaced), and the author should keep a complete copy. Manuscripts may be sent to any one of the four editors. All other communications to the editors should be addressed to the managing editor, L. J. Paige at the University of California, Los Angeles 24, California.

50 reprints per author of each article are furnished free of charge; additional copies may be obtained at cost in multiples of 50 .

The Pacific Journal of Mathematics is published quarterly, in March, June, September, and December. The price per volume (4 numbers) is $\$ 12.00$; single issues, $\$ 3.50$. Back numbers are available. Special price to individual faculty members of supporting institutions and to individual members of the American Mathematical Society: $\$ 4.00$ per volume; single issues, $\$ 1.25$.

Subscriptions, orders for back numbers, and changes of address should be sent to Pacific Journal of Mathematics, 103 Highland Boulevard, Berkeley 8, California.

Printed at Kokusai Bunken Insatsusha (International Academic Printing Co., Ltd.), No. 6, 2-chome, Fujimi-cho, Chiyoda-ku, Tokyo, Japan.

\section{PUBLISHED BY PACIFIC JOURNAL OF MATHEMATICS, A NON-PROFIT CORPORATION}

The Supporting Institutions listed above contribute to the cost of publication of this Journal, but they are not owners or publishers and have no responsibility for its content or policies.

Reprinted 1966 in the United States of America 


\section{Pacific Journal of Mathematics}

\section{Vol. 11, No. 4}

A. V. Balakrishnan, Prediction theory for Markoff processes . . . . . . . . . . 1171

Dallas O. Banks, Upper bounds for the eigenvalues of some vibrating systems . . . . 1183

A. Białynicki-Birula, On the field of rational functions of algebraic groups ...... 1205

Thomas Andrew Brown, Simple paths on convex polyhedra .............. 1211

L. Carlitz, Some congruences for the Bell polynomials . . . . . . . . . . . . 1215

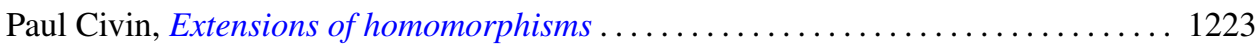

Paul Joseph Cohen and Milton Lees, Asymptotic decay of solutions of differential

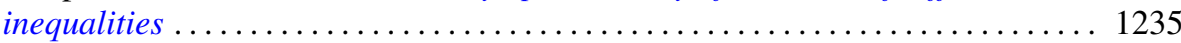

István Fáry, Self-intersection of a sphere on a complex quadric . . . . . . . . . . 1251

Walter Feit and John Griggs Thompson, Groups which have a faithful representation

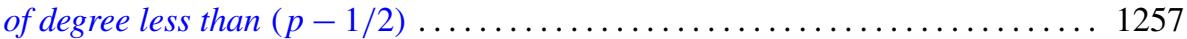

William James Firey, Mean cross-section measures of harmonic means of convex

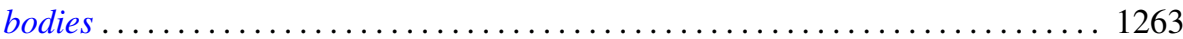

Avner Friedman, The wave equation for differential forms . . . . . . . . . . 1267

Bernard Russel Gelbaum and Jesus Gil De Lamadrid, Bases of tensor products of

Banach spaces ................................... 1281

Ronald Kay Getoor, Infinitely divisible probabilities on the hyperbolic plane . . . . 1287

Basil Gordon, Sequences in groups with distinct partial products . . . . . . . . . . . . 1309

Magnus R. Hestenes, Relative self-adjoint operators in Hilbert space . . . . . . . . . 1315

Fu Cheng Hsiang, On a theorem of Fejér ......................... 1359

John McCormick Irwin and Elbert A. Walker, On N-high subgroups of Abelian

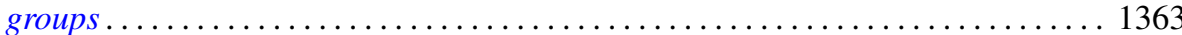

John McCormick Irwin, High subgroups of Abelian torsion groups . . . . . . . . . 1375

R. E. Johnson, Quotient rings of rings with zero singular ideal . . . . . . . . . . . 1385

David G. Kendall and John Leonard Mott, The asymptotic distribution of the time-to-escape for comets strongly bound to the solar system ...

Kurt Kreith, The spectrum of singular self-adjoint elliptic operators ....

Lionello Lombardi, The semicontinuity of the most general integral of the calculus of variations in non-parametric form ................................

Albert W. Marshall and Ingram Olkin, Game theoretic proof that Chebyshev inequalities are sharp

Wallace Smith Martindale, III, Primitive algebras with involution . . William H. Mills, Decomposition of holomorphs ..............

James Donald Monk, On the representation theory for cylindric algebras . . . . . . 1447

Shu-Teh Chen Moy, A note on generalizations of Shannon-McMillan theorem . . . . 1459

Donald Earl Myers, An imbedding space for Schwartz distributions . .

John R. Myhill, Category methods in recursion theory .........

Paul Adrian Nickel, On extremal properties for annular radial and circular slit mappings of bordered Riemann surfaces

Edward Scott O'Keefe, Primal clusters of two-element algebras . .

Nelson Onuchic, Applications of the topological method of Wazewski to certain

problems of asymptotic behavior in ordinary differential equations ...

Peter Perkins, A theorem on regular matrices................

Clinton M. Petty, Centroid surfaces .... 